\title{
TITLE:
}

\section{Electronic and structural properties of the oxygen vacancy in BaTiO3}

$\operatorname{AUTHOR}(\mathrm{S})$ :

Choi, Minseok; Oba, Fumiyasu; Tanaka, Isao

\section{CITATION:}

Choi, Minseok ... [et al]. Electronic and structural properties of the oxygen vacancy in BaTiO3. APPLIED PHYSICS LETTERS 2011, 98(17): 172901.

\section{ISSUE DATE:}

2011-04

URL:

http://hdl.handle.net/2433/160634

\section{RIGHT:}

Copyright 2011 American Institute of Physics. This article may be downloaded for personal use only. Any other use requires prior permission of the author and the American Institute of Physics. The following article appeared in APPLIED PHYSICS LETTERS 98, 172901 (2011) and may be found at http://link.aip.org/link/?apl/98/172901 


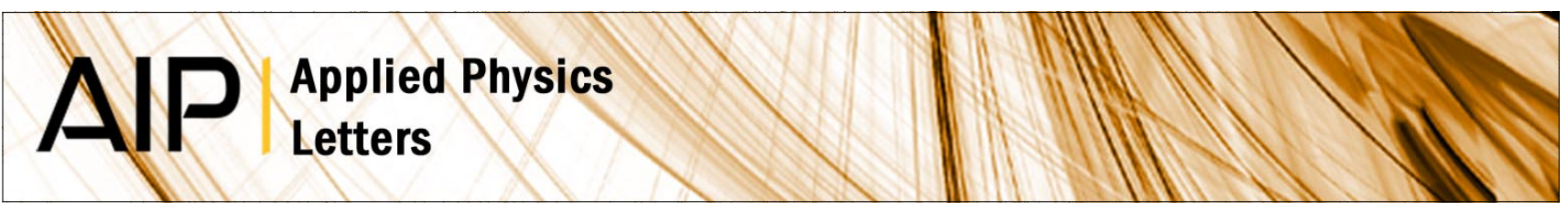

\section{Electronic and structural properties of the oxygen vacancy in BaTiO3}

Minseok Choi, Fumiyasu Oba, and Isao Tanaka

Citation: Appl. Phys. Lett. 98, 172901 (2011); doi: 10.1063/1.3583460

View online: http://dx.doi.org/10.1063/1.3583460

View Table of Contents: http://apl.aip.org/resource/1/APPLAB/v98/i17

Published by the American Institute of Physics.

\section{Related Articles}

Electron spin resonance signature of the oxygen vacancy in $\mathrm{HfO} 2$

Appl. Phys. Lett. 101, 102904 (2012)

Cationic point defects in $\mathrm{CuGaSe} 2$ from a structural perspective

Appl. Phys. Lett. 101, 101907 (2012)

Stable highly conductive $\mathrm{ZnO}$ via reduction of $\mathrm{Zn}$ vacancies

Appl. Phys. Lett. 101, 102101 (2012)

Engineering shallow spins in diamond with nitrogen delta-doping

Appl. Phys. Lett. 101, 082413 (2012)

Identifying a suitable passivation route for $\mathrm{Ge}$ interfaces

Appl. Phys. Lett. 101, 052903 (2012)

\section{Additional information on Appl. Phys. Lett.}

Journal Homepage: http://apl.aip.org/

Journal Information: http://apl.aip.org/about/about_the_journal

Top downloads: http://apl.aip.org/features/most_downloaded

Information for Authors: http://apl.aip.org/authors

\section{ADVERTISEMENT}

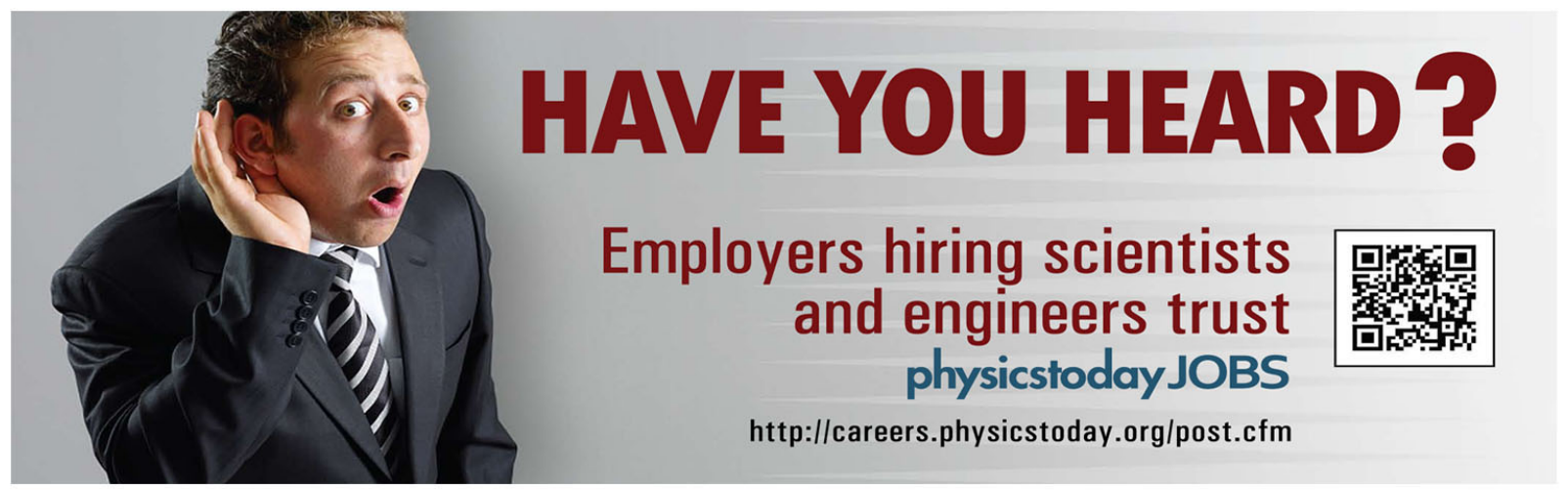




\title{
Electronic and structural properties of the oxygen vacancy in $\mathrm{BaTiO}_{3}$
}

\author{
Minseok Choi, ${ }^{1, a)}$ Fumiyasu Oba, ${ }^{1, b)}$ and Isao Tanaka ${ }^{1,2}$ \\ ${ }^{1}$ Department of Materials Science and Engineering, Kyoto University, Sakyo, Kyoto 606-8501, Japan \\ ${ }^{2}$ Nanostructures Research Laboratory, Japan Fine Ceramics Center, Atsuta, Nagoya 456-8587, Japan
}

(Received 16 February 2011; accepted 6 April 2011; published online 26 April 2011)

\begin{abstract}
The electronic and structural properties of the oxygen vacancy $\left(V_{\mathrm{O}}\right)$ in cubic $\mathrm{BaTiO}_{3}$ are studied using first-principles calculations based on a hybrid Hartree-Fock density functional approach. Our calculations identify the double shallow donor behavior of $V_{\mathrm{O}}$, indicating its contribution to the $n$-type conductivity. In addition, a metastable configuration is found, which shows an off-symmetric atomic structure around $V_{\mathrm{O}}$ in conjunction with deep localized electronic states in the band gap. Based on the identified characteristics of $V_{\mathrm{O}}$, the previous experimental and theoretical findings are explained. (C) 2011 American Institute of Physics. [doi:10.1063/1.3583460]
\end{abstract}

Perovskite titanates have been extensively investigated owing to its abundant physics and great applications. ${ }^{1-3}$ Among them, $\mathrm{BaTiO}_{3}$ is a prototypical ferroelectric and used for thin-film capacitors. ${ }^{1}$ Through lots of investigations, the native defect, especially, the oxygen vacancy $\left(V_{\mathrm{O}}\right)$ is known to significantly influence the physical properties of $\mathrm{BaTiO}_{3}$. It thus needs to clarify the structural and electronic properties of $V_{\mathrm{O}}$, but there exists controversy. Experimentally, $V_{\mathrm{O}}^{+}$was suggested to be stable in $n$-type specimens in wide range of temperature from electric conductivity measurements, ${ }^{4-6}$ whereas electron paramagnetic resonance (EPR) experiments have not clearly identified the physical feature of $V_{\mathrm{O}}^{+}$. Likewise, several first-principles studies have been conducted about $V_{\mathrm{O}}$ using the supercell approach with the local density approximation (LDA) or generalized gradient approximation (GGA) and the embedded-cluster approach with MP2. ${ }^{10-12}$ However, the results are scattered because of the different approximations, simulation models, and/or postprocesses applied to reduce errors, e.g., the severe band-gap error of the LDA and GGA.

In this Letter, our aim is to accurately illustrate the electronic and structural properties of $V_{\mathrm{O}}$ in cubic $\mathrm{BaTiO}_{3}$ using the Heyd-Scuseria-Ernzerhof (HSE06) hybrid functional. ${ }^{13,14}$ The use of HSE06 seems to be rationale, since this hybrid functional has accurately described defect physics in wide band-gap oxides. ${ }^{15-17}$ Through systematic calculations, we manifest that $V_{\mathrm{O}}$ plays a role as a double shallow donor under thermal equilibrium. Some experimental observations of $V_{\mathrm{O}}^{+}$are suggested to be responsible for the metastable configuration of $V_{\mathrm{O}}$ with a deep, localized electronic state or other causes.

The calculations were performed using the projector augmented-wave method ${ }^{18}$ as implemented in the VASP code. ${ }^{19}$ The standard exchange mixing containing $25 \%$ of Hartree-Fock and $75 \%$ of the Perdew-Burke-ErnzerhofGGA (Ref. 20) was employed in the HSE06 functional. The effects of spin polarization were considered for supercells with $V_{\mathrm{O}}$. The electronic wave functions were described using a plane wave basis set with an energy cutoff of $400 \mathrm{eV}$. A 135 -atom supercell and the $\Gamma$-only $k$-point sampling were used. The atomic coordinates were relaxed until

\footnotetext{
${ }^{a)}$ Electronic mail: choitheory@gmail.com.

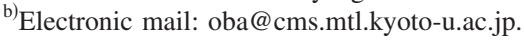

the Hellmann-Feynman force acting on each atom was reduced to less than $0.05 \mathrm{eV} \AA^{-1}$.

The formation energy of $V_{\mathrm{O}}$ is evaluated as

$$
\Delta E_{f}\left(V_{\mathrm{O}}^{q}\right)=E_{\mathrm{T}}\left(V_{\mathrm{O}}^{q}\right)-E_{\mathrm{T}}(\mathrm{H})+\mu_{\mathrm{O}}+q\left(E_{\mathrm{VBM}}+\varepsilon_{\mathrm{F}}\right),
$$

where $E_{\mathrm{T}}\left(V_{\mathrm{O}}^{q}\right)$ is the total energy of a supercell with one $V_{\mathrm{O}}$ in charge state $q$, and $E_{\mathrm{T}}(\mathrm{H})$ is the total energy of the host supercell. $\mu_{\mathrm{O}}$ is the oxygen chemical potential, varying between the O-rich limit $\left[\mu_{\mathrm{O}}=(1 / 2) \mu_{\mathrm{O}_{2} \text { (molecule) }}\right]$ and the O-poor limit $\left(\mu_{\mathrm{Ti}}=\mu_{\mathrm{Ti} \text { (bulk) }}\right.$ and $\left.\mu_{\mathrm{Ti}}+\mu_{\mathrm{O}}=\mu_{\mathrm{TiO}(\text { bulk })}\right)$. These equilibrium conditions are taken on the basis of previous considerations. ${ }^{21} E_{\mathrm{VBM}}$ and $\varepsilon_{\mathrm{F}}$ are the valence band maximum (VBM) with a potential alignment correction ${ }^{22}$ and the Fermi level measured from the VBM, respectively. As listed in Table I, HSE06 reproduces the lattice constant and band gap of $\mathrm{BaTiO}_{3}$ much better than GGA and GGA $+U$. However, the HSE06 band gap is still slightly smaller than the experimental value. To complement the small band-gap error, a post-correction was applied via a rigid shift in the conduction band minimum (CBM) and defect-induced states with $\mathrm{CB}$ characteristics. ${ }^{3,22}$

Figures 1(a) and 1(b) show the formation energy of $V_{\mathrm{O}}$ in $\mathrm{BaTiO}_{3}$ as a function of the Fermi level. $V_{\mathrm{O}}^{2+}$ is the most stable for most position of the Fermi level, and the formation energies for three charge states become comparable with each other when the Fermi level is close to the CBM. These characteristics indicate that $V_{\mathrm{O}}$ behaves as a double shallow donor, which releases two electrons. At the O-rich limit, the formation energy of $V_{\mathrm{O}}^{2+}$ when the Fermi level is located at the CBM, $\Delta E_{f}^{\mathrm{CBM}}\left(V_{\mathrm{O}}^{2+}\right)$, is $5.31 \mathrm{eV}$. The formation of $V_{\mathrm{O}}$ is therefore unlikely under O-rich conditions. Moving to the O-poor limit, the formation energy is lowered by $4.66 \mathrm{eV}$. A low $\Delta E_{f}^{\mathrm{CBM}}\left(V_{\mathrm{O}}^{2+}\right)$ value of $0.65 \mathrm{eV}$ indicates a substantial

TABLE I. Lattice constant $(a)$ and band gap $\left(E_{\mathrm{g}}\right)$ of cubic $\mathrm{BaTiO}_{3}$ obtained using the HSE06, GGA $+U\left(U_{\text {eff }}=4.36 \mathrm{eV}\right)$, (Refs. 3 and 23) and GGA Experimental values are also shown.

\begin{tabular}{lllll}
\hline \hline & HSE06 & GGA $+U$ & GGA & Experiment \\
\hline$a(\AA)$ & 3.993 & 4.064 & 4.036 & $3.996^{\mathrm{a}}$ \\
$E_{\mathrm{g}}(\mathrm{eV})$ & 3.11 & 2.14 & 1.67 & $3.4^{\mathrm{b}}$ \\
\hline \hline
\end{tabular}

${ }^{\mathrm{a}}$ Reference 24

${ }^{\mathrm{b}}$ Reference 10 
(a)

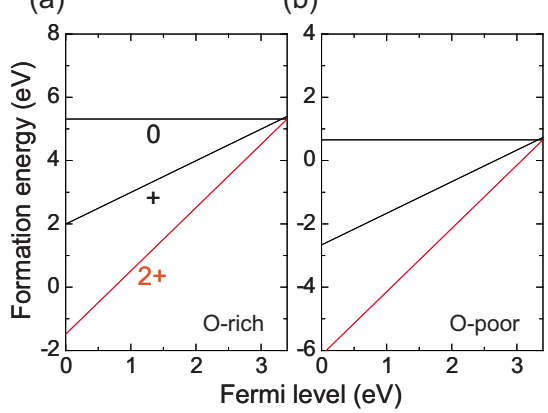

(c)

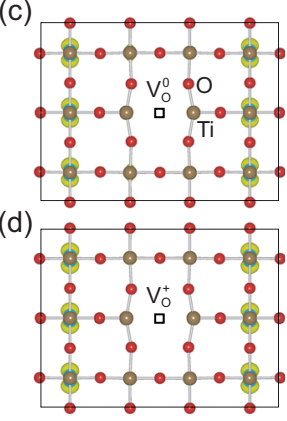

FIG. 1. (Color online) Formation energy of $V_{\mathrm{O}}$ in $\mathrm{BaTiO}_{3}$ as a function of the Fermi level under (a) the O-rich and (b) the O-poor limits. The charge states of $0,+$, and $2+$ correspond to the slopes. The squared wave functions $\left(\left|\psi_{\mathrm{e}}\right|^{2}\right)$ of the electron-occupied states in the conduction band for (c) $V_{\mathrm{O}}^{0}$ and (d) $V_{\mathrm{O}}^{+} \cdot\left|\psi_{\mathrm{e}}\right|^{2}$ are illustrated on the Ti-O plane including $V_{\mathrm{O}}$, which are the cross-sections of isosurfaces at $>0.007 \AA^{-3}$.

concentration of $V_{\mathrm{O}}$ under $\mathrm{O}$-poor conditions. As illustrated in Fig. 1(c), $V_{\mathrm{O}}^{0}$ creates a hydrogenic effective-mass electronic state occupied by two electrons. $V_{\mathrm{O}}^{+}$behaves very similarly, apart from the fact that the state is occupied by one electron in this case [Fig. 1(d)]. This feature is consistent with the formation-energy diagram, indicating nearly the same formation energies for $V_{\mathrm{O}}^{0}, V_{\mathrm{O}}^{+}$, and $V_{\mathrm{O}}^{2+}$ at the Fermi level near the CBM.

Additionally, we find a metastable configuration of $V_{\mathrm{O}}$ with an off-symmetric atomic structure. The metastable $V_{\mathrm{O}}$ possesses $C_{4 v}$ point-group symmetry and the distances between the two nearest $\mathrm{Ti}$ atoms and $V_{\mathrm{O}}$ are asymmetric: for $V_{\mathrm{O}}^{0}, 1.85 \AA$, and $2.47 \AA$, corresponding to the Ti displacements of $0.15 \AA$ (inward) and $0.47 \AA$ (outward) from their ideal positions, respectively. As shown in Fig. 2, the electron-occupied states at 0.28 and $0.66 \mathrm{eV}$ below the CBM are found in the case of the neutral charge state. The former is characterized by a host CBM-like orbital, while the latter, i.e., the deeper state, exhibits squared wave function $\left(\left|\psi_{\mathrm{e}}\right|^{2}\right)$ localized in the vicinity of $V_{\mathrm{O}}$. In the + charge state, an occupied localized state of $0.57 \mathrm{eV}$ below the CBM is created, similar to the deeper state in $V_{\mathrm{O}}^{0}$. However, it should be emphasized here that these configurations are energetically less favorable by $0.83 \mathrm{eV}$ for $V_{\mathrm{O}}^{0}$ and $1.19 \mathrm{eV}$ for $V_{\mathrm{O}}^{+}$than the most stable configurations shown in Fig. 1. ${ }^{25}$ Therefore, they are metastable and unlikely to form under thermal equilib-

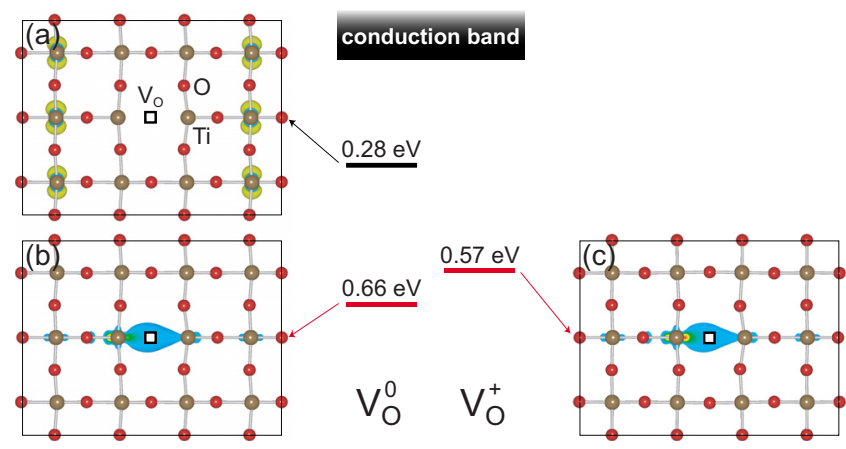

FIG. 2. (Color online) Electron-occupied single-particle states below the $\mathrm{CBM}$ and the corresponding $\left|\psi_{\mathrm{e}}\right|^{2}$ in metastable configurations of (a), (b) $V_{\mathrm{O}}^{0}$, and (c) $V_{\mathrm{O}}^{+}$. These states are for the majority spin component and any states are not found for the minority spin component. $\left|\psi_{\mathrm{e}}\right|^{2}$ are illustrated on the Ti-O plane including $V_{\mathrm{O}}$, which are the cross-sections of isosurfaces at $>0.007 \AA^{-3}$ for (a) and $>0.03 \AA^{-3}$ for (b) and (c). rium; however, they may form under specific growth conditions or treatments.

In previous supercell approaches using the LDA or GGA, $V_{\mathrm{O}}$ has been found to be a double shallow donor ${ }^{10,11}$ as we identified through the present hybrid functional calculations. Note, however, that the LDA and GGA in conjunction with the band-gap correction significantly overestimate $\Delta E_{f}$ (by $\sim 3 \mathrm{eV}$ ), compared with the present hybrid functional results. Similar pictures were recently found in other oxides. $^{15-17}$ In contrast to the supercell approaches, an embedded-cluster approach with MP2 suggested a localized characteristic, ${ }^{12}$ which is similar to the metastable configuration shown in Fig. 2. It is noteworthy that the LDA and GGA do not stabilize the localized configuration presumably due to the well-known delocalization error. The embeddedcluster approach cannot treat delocalized states and therefore it cannot predict the relative stability of the localized and delocalized configurations. Our results indeed show the metastability of the localized configuration with a sizable energy difference from the stable configuration.

On the basis of our results, we now discuss the experimental observations relevant to $V_{\mathrm{O}}$ in $\mathrm{BaTiO}_{3}$. In spite of the fact that many EPR measurements have been performed, the physical features and even existence of $V_{\mathrm{O}}^{+}$are unclear. $^{7-9}$ Several EPR measurements assigned their signals to a paramagnetic center, $\mathrm{Ti}^{3+}-V_{\mathrm{O}}^{2+}$, composed of $V_{\mathrm{O}}^{2+}$ with the nearest $\mathrm{Ti}^{4+}$ ion trapping one electron. Considering its total charge, this paramagnetic center corresponds to $V_{\mathrm{O}}^{+}$. However, the $\mathrm{g}$-factors assigned to this center are very much different from each other, and these are observed at very low temperature $(\sim 4 \mathrm{~K})$ to that higher than the Curie temperature $\left(>T_{C}\right.$ $\sim 390 \mathrm{~K})$. Therefore, the paramagnetic center is present in not only the tetragonal phase below the Curie temperature but also the cubic phase. Theoretically, it has been reported that $V_{\mathrm{O}}$ in the tetragonal and cubic phases has a similar characteristic in its electronic structure. ${ }^{26} \mathrm{~A}$ recent experiment suggested another $\mathrm{Ti}^{3+}-V_{\mathrm{O}}^{2+}$ center in thin films. ${ }^{8}$ Laguta et $a l .{ }^{8}$ claimed that the g-factor comes from the occupied $e_{\mathrm{g}}\left(d_{3 z^{2}-r^{2}}\right)$ state as the ground state of $V_{\mathrm{O}}^{+}$, as found in an embedded-cluster study, ${ }^{12}$ and thereby their EPR signal originates from $V_{\mathrm{O}}^{+}$. Another EPR experiment predicted that $V_{\mathrm{O}}^{+}$possesses $C_{4 v}$ symmetry and its ground state becomes $t_{2 \mathrm{~g}}$ if an acceptor-like impurity is located nearby it. ${ }^{9}$

As seen in Figs. 1(c) and 1(d), however, $V_{\mathrm{O}}$ has spatially delocalized electrons under thermal equilibrium. Thus, the stable $V_{\mathrm{O}}^{+}$configuration cannot explain the paramagnetic centers mentioned above. Differently, the metastable configuration of $V_{\mathrm{O}}^{+}$has $C_{4 v}$ symmetry and an in-gap state with a $d_{3 z^{2}-r^{2}}$ and $d_{x^{2}-y^{2}}$ mixed characteristic $\left|\psi_{\mathrm{e}}\right|^{2}$, mainly localized in the vicinity of $V_{\mathrm{O}}$ and one of its first nearest Ti atoms [Fig. 2(c)]: the electron-population of $\left|\psi_{\mathrm{e}}\right|^{2}$ on the first nearest Ti site is three times higher than that on the second nearest $\mathrm{Ti}$ site. The metastable $V_{\mathrm{O}}^{+}$is therefore possibly responsible for the observed EPR signals. In fact, the presence of $\mathrm{Ti}^{3+}$ $-V_{\mathrm{O}}^{2+}$ centers has been suggested in polycrystalline films synthesized using rf sputter deposition, ${ }^{8}$ multidomain crystals after reduction under $\mathrm{H}_{2}$ atmosphere, ${ }^{9}$ and polycrystals obtained by thermal decomposition of $\operatorname{BaTiO}\left(\mathrm{C}_{2} \mathrm{O}_{4}\right)_{2} \cdot 4 \mathrm{H}_{2} \mathrm{O}$ precursors. ${ }^{7}$ On the other hand, there exists no report on the observation of the $\mathrm{Ti}^{3+}-V_{\mathrm{O}}^{2+}$ center in single crystals. Laguta et $a .^{8}{ }^{8}$ mentioned that the observation of the paramagnetic center in thin films but not in bulk crystals may be attributed 
to the specific conditions under which the thin films are processed. These lead us to a suggestion that the EPR signal of $V_{\mathrm{O}}^{+}$results from the metastable configuration of $V_{\mathrm{O}}$.

According to the $\mathrm{O}_{2}$ partial pressure dependence of the electric conductivity for polycrystalline $\mathrm{BaTiO}_{3}$ specimens, $V_{\mathrm{O}}$ is fully ionized at high temperatures above $\sim 873 \mathrm{~K} .{ }^{5} \mathrm{An}$ estimated ionization energy for $V_{\mathrm{O}}^{+} \rightarrow V_{\mathrm{O}}^{2+}$ is $\sim 0.5 \mathrm{eV}$, while for $V_{\mathrm{O}}^{0} \rightarrow V_{\mathrm{O}}^{+}$, the ionization energy is only a few hundredths of electron volt. ${ }^{6}$ The experimentally assigned high ionization energy of $\sim 0.5 \mathrm{eV}$ is incompatible with the double shallow donor nature of $V_{\mathrm{O}}$, predicted in our study. Therefore, the deep donor level may be responsible not for isolated $V_{\mathrm{O}}$ but for other defects, complexes, or impurities.

Very recently, a relaxorlike state, which is typically caused by the formation of local polar nanoregions above paraelectric $\rightarrow$ ferroelectric transition or the Burns temperatures, was found for the high-temperature paraelectric cubic phase in $\mathrm{BaTiO}_{3}$ single crystals. ${ }^{27}$ It is often understood that the observed relaxor state in nominally pure samples can be related to some defects that induce the inversion symmetry breaking. According to our results, thermodynamically the most stable configuration of $V_{\mathrm{O}}$ is symmetric, and thus is unlikely to be a cause. However, the metastable $V_{O}$ breaks the inversion symmetry, and thereby may produce the polarization when it can form with a high enough concentration. Lately, another perovskite titanate, $\mathrm{SrTiO}_{3}$, was reported to exhibit a ferroelectric state, in the nonstoichiometric bulk and thin films with O-deficiency ${ }^{28}$ and a composition of $\mathrm{Sr} / \mathrm{Ti}<1 .{ }^{29}$ It was theoretically proposed that $\mathrm{Ti}$ antisite defects off-centered from the $\mathrm{Sr}$ site can lead to the polarization owing to their local electric dipoles and low formation energies under Ti-rich conditions. ${ }^{3}$ Analogically, one can speculate that $\mathrm{Ti}$ antisites, composed of a $\mathrm{Ti}$ atom off-centered from the $\mathrm{Ba}$ site, are also likely causes of the observed relaxor-like state in $\mathrm{BaTiO}_{3}$.

To summarize, we have investigated $V_{\mathrm{O}}$ in $\mathrm{BaTiO}_{3}$ using the hybrid density-functional calculations. $V_{\mathrm{O}}$ is a double shallow donor under thermal equilibrium and hence can contribute to the $n$-type conductivity. A metastable asymmetric atomic-configuration also exists and its formation probably depends on specific growth conditions. The metastable configuration can be responsible for the observed EPR signals in non-single crystalline $\mathrm{BaTiO}_{3}$.

This work was supported by Grants-in-Aid for Scientific Research (A), Young Scientists (B), Scientific Research on Priority Areas (No. 474), and a Global COE Program.

\footnotetext{
${ }^{1}$ Physics of Ferroelectrics: A Modern Perspective, Topics in Applied Physics Vol. 105, edited by K. Rabe, C. H. Ahn, and J.-M. Triscone (Springer, Berlin, 2007).
}

${ }^{2}$ M. Choi, D. H. Kim, and C.-H. Park, J. Korean Phys. Soc. 49, 481 (2006).

${ }^{3}$ M. Choi, F. Oba, and I. Tanaka, Phys. Rev. Lett. 103, 185502 (2009).

${ }^{4}$ N. H. Chan, R. K. Sharm, and D. M. Smyth, J. Am. Ceram. Soc. 64, 556 (1981).

${ }^{5}$ D. M. Smyth, Prog. Solid State Chem. 15, 145 (1984).

${ }^{6}$ I. P. Raevski, S. M. Maksimov, A. V. Fisenko, S. A. Prosandeyev, I. A. Osipenko, and P. F. Tarasenko, J. Phys.: Condens. Matter 10, 8015 (1998).

${ }^{7}$ T. R. N. Kutty, P. Murugaraj, and N. S. Gajbhiye, Mater. Lett. 2, 396 (1984)

${ }^{8}$ V. V. Laguta, A. M. Slipenyuk, I. P. Bykov, M. D. Glinchuk, M. Maglione, D. Michau, J. Rosa, and L. Jastrabik, Appl. Phys. Lett. 87, 022903 (2005)

${ }^{9}$ R. Scharfschwerdt, A. Mazur, O. F. Schirmer, H. Hesse, and S. Mendricks, Phys. Rev. B 54, 15284 (1996).

${ }^{10} \mathrm{P}$. Erhart and K. Albe, J. Appl. Phys. 102, 084111 (2007).

${ }^{11}$ Y. Iwazaki, T. Suzuki, and S. Tsuneyuki, J. Appl. Phys. 108, 083705 (2010).

${ }^{12}$ H. Donnerberg and A. Birkholz, J. Phys.: Condens. Matter 12, 8239 (2000).

${ }^{13}$ J. Heyd, G. E. Scuseria, and M. Ernzerhof, J. Chem. Phys. 118, 8207 (2003); 124, 219906 (2006).

${ }^{14}$ A. V. Krukau, O. A. Vydrov, A. F. Izmaylov, and G. E. Scuseria, J. Chem. Phys. 125, 224106 (2006).

${ }^{15}$ F. Oba, A. Togo, I. Tanaka, J. Paier, and G. Kresse, Phys. Rev. B 77 245202 (2008).

${ }^{16}$ A. Janotti, J. B. Varley, P. Rinke, N. Umezawa, G. Kresse, and C. G. Van de Walle, Phys. Rev. B 81, 085212 (2010).

${ }^{17}$ P. Ágoston, K. Albe, R. M. Nieminen, and M. J. Puska, Phys. Rev. Lett. 103, 245501 (2009).

${ }^{18}$ P. E. Blöchl, Phys. Rev. B 50, 17953 (1994).

${ }^{19}$ G. Kresse and J. Hafner, Phys. Rev. B 48, 13115 (1993); G. Kresse and J. Furthmüller, ibid. 54, 11169 (1996); G. Kresse and D. Joubert, ibid. 59, 1758 (1999).

${ }^{20}$ J. P. Perdew, K. Burke, and M. Ernzerhof, Phys. Rev. Lett. 77, 3865 (1996).

${ }^{21}$ H.-S. Lee, T. Mizoguchi, T. Yamamoto, S.-J. L. Kang, and Y. Ikuhara, Acta Mater. 55, 6535 (2007)

${ }^{22}$ M. Choi, F. Oba, and I. Tanaka, Phys. Rev. B 78, 014115 (2008).

${ }^{23}$ S. L. Dudarev, G. A. Botton, S. Y. Savrasov, C. J. Humphreys, and A. P. Sutton, Phys. Rev. B 57, 1505 (1998).

${ }^{24}$ S. Miyake and R. Ueda, J. Phys. Soc. Jpn. 2, 93 (1947).

${ }^{25}$ The total energy of metastable $V_{\mathrm{O}}$ is lower than that of the most stable $V_{\mathrm{O}}$ shown in Fig. 1. However, the symmetry breaking toward $C_{4 v}$, i.e., tetragonal-like distortion also lowers the total energy of the perfect crystal cell. It seems that this results from the fact that the low-temperature tetragonal phase is more stable than the high-temperature cubic phase. To compensate the energy gain associated with the tetragonal-like distortion, we used the distorted perfect crystal for the evaluation of the formation energy of metastable $V_{\mathrm{O}}$. Note that another configuration exists with the $C_{4 v}$ symmetry, which shows no in-gap electron localized state as in the case of the stable $V_{\mathrm{O}}$. This configuration is more stable than the metastable $V_{\mathrm{O}}$ by $0.19 \mathrm{eV}$ and $0.20 \mathrm{eV}$ for the neutral and + charge states, respectively, supporting our conclusion that $V_{\mathrm{O}}$ acts as a shallow double donor.

${ }^{26}$ Z. Alahmed and H. Fu, Phys. Rev. B 76, 224101 (2007).

${ }^{27}$ E. Dul'kin, J. Petzelt, S. Kamba, E. Mojaev, and M. Roth, Appl. Phys. Lett. 97, 032903 (2010).

${ }^{28}$ Y. S. Kim, D. J. Kim, T. H. Kim, T. W. Noh, J. S. Choi, B. H. Park, and J.-G. Yoon, Appl. Phys. Lett. 91, 042908 (2007).

${ }^{29}$ H. W. Jang, A. Kumar, S. Denev, M. D. Biegalski, P. Maksymovych, C. W. Bark, C. T. Nelson, C. M. Folkman, S. H. Baek, and N. Balke, Phys. Rev. Lett. 104, 197601 (2010). 